\title{
Factores asociados a bajo rendimiento académico en estudiantes de medicina humana de la Universidad Ricardo Palma durante el período agosto-diciembre del 2020
}

\author{
Factors associated with low academic performance in human medicine students at Ricardo Palma \\ University during the period August-December 2020
}

\author{
Jennifer M. Otero-Zapata', \\ Sonia Indacochea-Cáceda², \\ Alonso Soto-Tarazona ${ }^{3}$, \\ Jhony De la Cruz-Vargas ${ }^{4}$
}

\author{
Otero-Zapata JM, Indacochea-Cáceda S, Soto-Tarazona A, De la \\ Cruz-Vargas J. Factores asociados a bajo rendimiento académico en \\ estudiantes de medicina humana de la Universidad Ricardo Palma \\ durante el período agosto-diciembre del 2020. Rev Soc Peru Med \\ Interna. 2021;34(3):93-99 \\ https://doi.org/10.36393/spmi.v34i3.608
}

RESUMEN

Introducción: El bajo rendimiento académico a nivel universitario es un tema que llena de frustración al alumno porque es un obstáculo para su vida profesional y esto se puede deber a diversos factores.

Objetivo: Determinar los factores asociados al bajo rendimiento académico en los estudiantes de medicina humana. Material y Métodos: Se llevó a cabo un estudio observacional, analítico de corte transversal, en los estudiantes de medicina humana de la Universidad Ricardo Palma, Lima, Perú, durante el periodo agosto-diciembre del 2020. El estudio se hizo mediante un cuestionario de 40 preguntas sobre características sociodemográficas, bajo rendimiento académico, trastorno por déficit de atención e hiperactividad (TDAH), ansiedad, depresión y consumo de tabaco y alcohol. El análisis bivariado se realizó mediante pruebas estadísticas Chi-cuadrado o Fisher, según el caso. El análisis multivariado usó modelos lineales generalizados con familia de distribución de Poisson y función de enlace logística con un IC del 95\%. Se consideró p <0,05 como significativo. Resultados: De los 273 estudiantes encuestados, el 74,4\% fue de sexo femenino. Se encontró una prevalencia de bajo rendimiento académico del 7I,4\% en alguna asignatura cursada durante el ciclo 2020-II. Se evidenció que los factores relacionados a bajo rendimiento académico fueron la edad, RP I,09 (IC 95\% I,07-I, I 2, P<0,00 I) y aquellos con sintomatología de TDAH, RP de I,22 (IC 95\% I,07-I,4, $\mathrm{p}=0,003)$. Conclusiones: Los estudiantes de medicina humana presentaron una alta prevalencia de bajo rendimiento académico, $y$ en asociación significativa al tener mayor edad y presentar sintomatología de TDAH.

Palabras claves: Rendimiento académico, estudiantes de medicina, trastorno por déficit de atención con hiperactividad, ansiedad, depresión, etanol, fumar. (DeCS)

Bachiller en medicina humana. Universidad Ricardo Palma, Lima, Perú.

2 Médico internista. Hospital Nacional Edgardo Rebagliati Martins. Docente e investigadora del Instituto de Investigaciones Biomédicos, Universidad Ricardo Palma, Lima, Perú.

3 Médico internista Hospital Nacional Hipólito Unanue. Docente e investigador del Instituto de Investigaciones Biomédicos, Universidad Ricardo Palma, Lima, Perú.

4 Médico internista. Docente e investigador del Instituto de Investigaciones Biomédicos, Universidad Ricardo Palma, Lima, Perú. 


\begin{abstract}
Introduction: Poor academic performance at the university level is an issue that fills the student with frustration because it is an obstacle to their professional life and this may be due to various factors. Objective:To determine the factors associated with low academic performance in human medicine students. Material and Methods: An observational, analytical cross-sectional study was carried out in human medicine students at Ricardo Palma University, Lima, Peru, during the period August-December 2020. The study was conducted using a 40-question questionnaire on sociodemographic characteristics, poor academic performance, attention deficit hyperactivity disorder (ADHD), anxiety, depression, and tobacco and alcohol use. Bivariate analysis was performed using Chisquare or Fisher statistical tests, as the case may be. Multivariate analysis used generalized linear models with Poisson distribution family and logistic link function with a $95 \% \mathrm{Cl}$. P<0,05 was considered significant. Results: Of the 273 students surveyed, 74,4\% were female. A prevalence of low academic performance of $71.4 \%$ was found in some subject taken during the 2020-II cycle. It was evidenced that the factors related to low academic performance were age, PR I,09 (95\% Cl I,07-I, I2, $p<0.001)$ and those with symptoms of ADHD, PR of I,22 (95\% CI I,07-I,4, p=0,003).

Conclusions: Human medicine students presented a high prevalence of low academic performance, and in significant association to be older and present symptoms of ADHD.
\end{abstract}

Key words:Academic performance, students, ADHD, anxiety, depression, ethanol, smoking. (MESH)

\section{INTRODUCCIÓN}

El bajo rendimiento académico es una constante preocupación para aquellos estudiantes que quieren forjar un mejor futuro para ellos. Ahora, añadirle a esta situación el hecho de estudiar una carrera tan demandante, como lo es medicina humana, deviene en una frustración constante al no lograr las metas trazadas.

En la bibliografía se nos pone en contexto algunos elementos que podrían originar esta situación, como lo es el trastorno de déficit de atención e hiperactividad (TDAH), la depresión, la ansiedad, el consumo de drogas, entre otros aspectos. $^{1-4}$

El trastorno por déficit de atención e hiperactividad (TDAH) es un trastorno del neurodesarrollo definido por niveles problemáticos de inatención, desorganización y/o hiperactividad-impulsividad. ${ }^{5}$ La depresión es un estado de tristeza persistente tanto en tiempo como en intensidad, que interfiere con las actividades del individuo, produciendo pérdida de interés o de placer en estas. ${ }^{6}$ En la ansiedad intervienen características de angustia y nerviosismo excesivos y, a su vez, alteraciones conductuales asociadas. ${ }^{3}$ Estos componentes podrían llevar a una indebida concentración para avanzar en los estudios, produciendo limitantes académicas y, a la vez, generar la sensación de insatisfacción personal a nivel profesional, lo que puede acentuar la sintomatología de los trastornos ya mencionados y convertirse en un círculo vicioso.

Por ende, se busca saber cuáles son los factores que podrían limitar las capacidades, reflejado en el bajo rendimiento, de los alumnos para obtener sus objetivos.

\section{MATERIAL Y MÉTODOS}

Tipo y diseño. Estudio de tipo observacional, analítico, de corte transversal.

Población y Muestra. La población de estudio estuvo conformada por los estudiantes de medicina humana de los ciclos VI hasta el internado médico de la Universidad Ricardo Palma, Lima, durante el periodo agosto-diciembre del 2020 (ciclo 2020-II). Para calcular el tamaño muestral se utilizó el programa Epidat, donde se usó la frecuencia con el factor $23,9 \%$ y la frecuencia sin el factor de $45 \%$, una razón entre tamaños muestrales de $3,02 \%$, un nivel de confianza de $95 \%$ y un poder estadístico de $90 \%$, obteniéndose como tamaño de muestra 274 estudiantes.

Variables e instrumentos. Se aplicó un cuestionario dividido en cuatro partes.

La primera parte fue tomada de la encuesta validada de una tesis realizada en Trujillo, en el año 2019' ${ }^{1}$, donde se encuentra la variable de bajo rendimiento según la calificación de la Universidad Ricardo Palma. Así: aprobado, $\geq 11$ y desaprobado, $<11$ (bajo rendimiento académico), determinado por el Sistema de Calificaciones del Perú ${ }^{7}$ ), para las asignaturas del ciclo 2020-II.

La segunda parte constó del cuestionario autoinformado de cribado de TDAH del adulto versión 1.1 (ASRS v1.1) ${ }^{8}$ de la OMS, compuesta por 6 ítems que evalúan síntomas de TDAH. Sintomatología compatible con TDAH $\geq 4$ cuadros sombreados.

La tercera parte fue la Escala de Ansiedad y Depresión de Goldberg (EADG) ${ }^{9}$, que tiene 18 ítems divididos en 9 preguntas correspondientes a cada subescala. Sintomatología compatible con ansiedad $\geq 4$ puntos. Sintomatología compatible con depresión $\geq 2$ puntos.

Finalmente, la Encuesta de las Naciones Unidas sobre el consumo de drogas ${ }^{10}$, que incluía tabaco y alcohol, con 8 ítems, donde se obtuvo el autorreporte del estudiante.

Procedimientos. Se recolectó la información mediante el uso de una encuesta virtual, creada en Google Forms, la cual fue enviada a través de los correos electrónicos de los estudiantes incluidos en la presente investigación, adjuntando a su vez el consentimiento informado en formato Word, con el cual debían estar de acuerdo para continuar con el llenado de la encuesta. La investigadora (JMOZ) estuvo disponible vía remota durante la resolución del cuestionario. para solucionar cualquier duda durante el llenado. La duración promedio de llenado del cuestionario fue entre 5 a 10 minutos.

Análisis Estadístico. La información obtenida se registró 
en una hoja de cálculo de Microsoft Excel, y para detectar el registro de información errónea, se sometió las encuestas a un proceso de control de calidad que consistía en seleccionar aleatoriamente diez fichas de recolección de datos y se corroboró con los datos registrados en la hoja de cálculo de Excel.

Las variables cualitativas fueron codificadas en números para facilitar su exportación al programa estadístico STATA v.14,0. En el análisis descriptivo, las variables cuantitativas fueron descritas en términos de tendencia central y dispersión según su normalidad, y las variables cualitativas en frecuencias absolutas y relativas.

Para identificar una asociación entre variables se utilizó el estadístico Chi-cuadrado de independencia. Además, se calculó RP con sus respectivos intervalos de confianza. Todas las pruebas de hipótesis se aplicaron utilizando un nivel de significancia de $\mathrm{p}<0,05$. Para el análisis multivariado se incluyeron solo las variables con valores p significativos, además de la variable dependiente. Para obtener la medida de asociación correspondiente, se usaron modelos lineales generalizados con familia de distribución de Poisson y función de enlace logística con un intervalo de confianza del 95\%.

Aspectos éticos. Se contó con la aprobación del Comité de Ética e Investigación del Instituto de Investigación en Ciencias Biomédicas (INICIB) y Facultad de Medicina Humana de la Universidad Ricardo Palma. Se respetó la confidencialidad de los datos; así, no se publicó dato alguno que permitiera identificar a los participantes.

\section{RESULTADOS}

Se encuestó a 273 estudiantes, de los cuales el 74,4\% fueron de sexo femenino. Las edades se encontraron en un rango de 17 a 32 años. Los ciclos y el número de estudiantes encuestados fueron: $6^{\circ}$ (16 estudiantes), $7^{\circ}$ (23 estudiantes), $8^{\circ}$ (29 estudiantes), $9^{\circ}$ (32 estudiantes), $10^{\circ}$ (34 estudiantes), $11^{\circ}$ ( 26 estudiantes), $12^{\circ}$ (44 estudiantes) e internado médico (69 estudiantes).

El 92,3\% de estudiantes usó algún método de estudio; vivía con su familia el $88,6 \%$; y, los que solo estudiaban sin trabajar, $85,3 \%$. Con respecto a la sintomatología de TDAH se obtuvo en $27,5 \%$, de ansiedad en $75,4 \%$ y depresión $78,8 \%$ de los estudiantes. Mientras que el consumo de drogas se vio en $36,6 \%$, de las cuales, el tabaco estuvo presente en $9,2 \%$ y alcohol en $27,5 \%$. Ver tabla 1 .

La frecuencia de bajo rendimiento académico en alguna asignatura cursada en el periodo 2020 -II fue de $71,4 \%$ $(\mathrm{n}=195)$.

Respecto a los test de cribado de aspectos psicológicos utilizados en este estudio, los estudiantes con sintomatología compatible con TDAH fue 27,5\% ( $n=75)$, con ansiedad $75,4 \%(n=214)$ y depresión 78,8\% $(n=215)$. Ver figura 1 . En lo que corresponde al consumo de drogas, el 63,4\% $(n=173)$ negó consumo alguno, mientras que el 36,6\% lo afirmó, tabaco $9,2 \%(n=25)$ y alcohol $27.5 \%(n=75)$.

En la tabla 2 se observa el análisis bivariado, donde se asoció las características de la población con el bajo rendimiento académico. Se encuentra asociación significativa con la edad $(\mathrm{p}<0,001)$, siendo la mediana 25 años. Respecto al ciclo actual se obtuvo un valor de $\mathrm{p}$ de 0,001 , con la mediana de $11^{\circ}$ ciclo. También, aquellos alumnos que estudiaban y trabajaban a la vez, el $90 \%$ presentó bajo rendimiento académico. A su vez, el TDAH se presentó en el $85,3 \%$ de los estudiantes con la ya mencionada deficiencia académica, mientras que, en el ámbito del consumo de drogas, el fumar tabaco se asoció con un valor de $\mathrm{p}<0,006$ y el $88 \%$ de los estudiantes que lo hizo presentó bajo rendimiento académico. Ver tabla 2.

En esta tabla se observa el análisis multivariado, tras realizar los ajustes en las variables edad, ciclo actual, estudia o trabaja, TDAH y Fuma, se halló que, los estudiantes son más propensos al bajo rendimiento en promedio en la edad de 25 años, con un RP 1.09 (IC 95\% 1.07-1.12, P<0.001), y en aquellos que padecen de TDAH presentan un RP de 1.22 (IC 95\% 1.07-1.4, $\mathrm{P}=0.003$ ).

Tabla I. Características sociodemográficas de la población del estudio

\begin{tabular}{|c|c|c|}
\hline \multicolumn{3}{|l|}{ Sexo } \\
\hline Femenino & 203 & $74,4 \%$ \\
\hline Masculino & 70 & $25,6 \%$ \\
\hline Edad (años) (mediana) & 24 & $\begin{array}{l}\text { rango intercuartílico: } \\
22-26\end{array}$ \\
\hline Ciclo actual (mediana) & II & $\begin{array}{l}\text { rango intercuartílico: } \\
9-13\end{array}$ \\
\hline \multicolumn{3}{|l|}{$\begin{array}{l}\text { Uso de método de } \\
\text { estudio }\end{array}$} \\
\hline Sí & 252 & $92,3 \%$ \\
\hline No & 21 & $7,7 \%$ \\
\hline \multicolumn{3}{|l|}{ Estudia o trabaja } \\
\hline Solo estudia & 233 & $85,3 \%$ \\
\hline Estudia y trabaja & 40 & $14,7 \%$ \\
\hline \multicolumn{3}{|l|}{ Vive con la familia o solo } \\
\hline Con la familia & 242 & $88,6 \%$ \\
\hline Solo & 31 & $1 \mathrm{I}, 4 \%$ \\
\hline
\end{tabular}

Figura I. Test de cribado de aspectos psicológicos en la población de estudio durante el ciclo 2020-II.

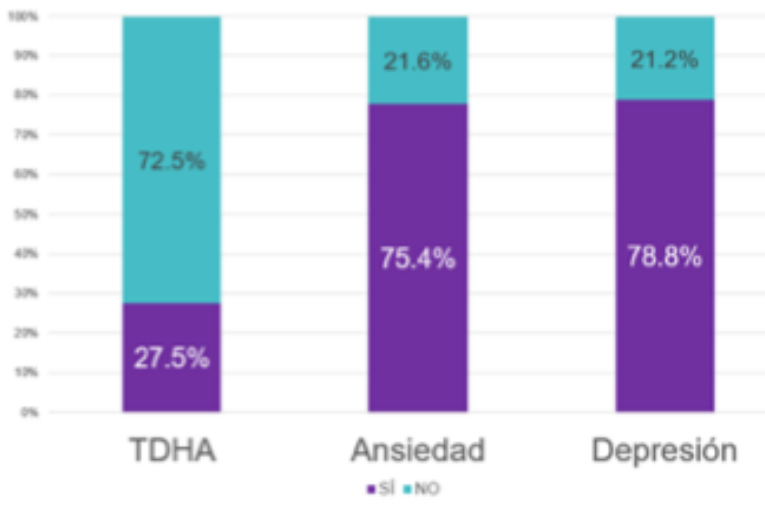


Tabla 2. Análisis bivariado de los factores asociados a bajo rendimiento académico.

\begin{tabular}{|c|c|c|c|c|c|}
\hline & \multicolumn{2}{|c|}{ Bajo rendimiento académico } & \multirow[t]{2}{*}{ Total } & \multirow[t]{2}{*}{ RP (IC 95\%) } & \multirow[t]{2}{*}{$\mathrm{P}$} \\
\hline & Sí (N=195) & No $(N=78)$ & & & \\
\hline Sexo & & & & & 0,763 \\
\hline Masculino & $21(30,0 \%)$ & $49(70,0 \%)$ & 70 & $0,98(0,8 I-I, I 6)$ & \\
\hline Femenino & | 46 (7|,9\%) & $57(28,1 \%)$ & 203 & Ref. & \\
\hline Edad (años) & $25(23-27)$ & $22(2 \mid-24)$ & $24(22-26)$ & $1,10(1,07-1,13)^{*}$ & $<0,001$ \\
\hline Ciclo actual & II (9-13) & $9(8-11)$ & II (9-13) & $1.06(1.02-1.1)^{*}$ & 0,001 \\
\hline Uso de métodos de estudio & & & & & 1,000 \\
\hline No & I5 (7I,4\%) & $6(28,6 \%)$ & 21 & $I, 00(0,76-I, 32)$ & \\
\hline Sí & I 80 (7I,4\%) & $72(28,6 \%)$ & 252 & Ref. & \\
\hline Estudia o trabaja & & & & & $<0,00$ I \\
\hline Estudia y trabaja & $36(90,0 \%)$ & $4(10,0 \%)$ & 40 & I,3I (I,I5-I,5I) & \\
\hline Solo estudia & $159(68.2 \%)$ & 74 (31.8\%) & 233 & Ref. & \\
\hline Vive con familia o solo & & & & & 0,157 \\
\hline Solo & $25(80,7 \%)$ & $6(19,3 \%)$ & 31 & I, I4 (0,94-I,40) & \\
\hline Familia & 170 (70,3\%) & $72(29,7 \%)$ & 242 & Ref. & \\
\hline TDAH & & & & & $<0,00$ I \\
\hline Sí & $64(85,3 \%)$ & II (I4,70\%) & 75 & I,28 (I,I2-I,50) & \\
\hline No & |3| (66,2\%) & $67(33,8 \%)$ & 198 & Ref. & \\
\hline Ansiedad & & & & & 0,133 \\
\hline Sí & I 58 (73,8\%) & $56(26,2 \%)$ & 214 & I,I 8 (0,95-I,45) & \\
\hline No & 37 (62,7\%) & $22(37,3 \%)$ & 59 & Ref. & \\
\hline Depresión & & & & & 0,373 \\
\hline Sí & I5I (70,2\%) & $64(29,8 \%)$ & 215 & $0,92(0,79-I, 10)$ & \\
\hline No & 44 (75,9\%) & 14 (24, I\%) & 58 & Ref. & \\
\hline Consumo de drogas & & & & & 0,906 \\
\hline Sí & 7I (7I,0\%) & $28(28,0 \%)$ & 100 & I $(0,84-I, 20)$ & \\
\hline No & I 24 (7I,7\%) & $49(28,3 \%)$ & 173 & Ref. & \\
\hline Tabaco & & & & & 0,006 \\
\hline Sí & $22(88,0 \%)$ & $3(12,0 \%)$ & 25 & I,26 (I,06-I,50) & \\
\hline No & $173(69,8 \%)$ & $75(30,2 \%)$ & 248 & Ref. & \\
\hline Alcohol & & & & & 0,865 \\
\hline Sí & $53(70,7 \%)$ & $22(29,3 \%)$ & 75 & $0,98(0,83-I, 16)$ & \\
\hline No & | 42 (7|,7\%) & $56(28,3 \%)$ & 198 & Ref. & \\
\hline
\end{tabular}

*Mediana y rango intercuartílico

\section{DISCUSIÓN}

En el presente estudio, podemos observar un gran porcentaje de estudiantes del $6^{\circ}$ al $14^{\circ}$ ciclo con bajo rendimiento académico en alguna asignatura cursada en el periodo 2020-II, siendo el 71,4\% que ha reprobado algún curso. En la Facultad de Medicina Humana, a partir del $6^{\circ}$ ciclo se inician las prácticas hospitalarias y al no poder acudir a estas, se ha visto la necesidad de ser reemplazadas por clases virtuales, donde la interacción médico-paciente como conocíamos ya no se realiza más, y los estudiantes que están en formación carecen en distintas medidas de esta experiencia, lo cual se ve reflejado en el rendimiento académico. He de añadir también que se podría plantear como sesgo de selección que los alumnos que más interés han tenido en responder la encuesta son aquellos que precisamente les importa el bajo rendimiento que presentan y que se refleja en las tasas altas de bajo rendimiento académico obtenidas.

Un artículo, elaborado en la Pontifica Universidad Católica de Ecuador, refirió que el 65,1\% de los encuestados en la Escuela de Psicología también presentó bajo rendimiento académico. Otro estudio, realizado en Trujillo, nos mostró una frecuencia del $45,9 \%$ de bajo rendimiento en estudiantes de medicina humana. ${ }^{1}$

Enlosestudiantesuniversitariosyacenmásresponsabilidades en diversos ámbitos $\mathrm{y}$, en el aspecto académico son más exigentes aún; pero, muchas veces, estas exigencias no 
se ven realizadas por diversos factores, como la edad, en la presente investigación, donde se observó que mientras más edad se tiene, más bajo rendimiento se obtiene. Brito y Palacio ${ }^{11}$ hallaron que a menor edad hubo mejor rendimiento académico y a mayor edad este rendimiento se vio disminuido. En la tesis de Roque también se reafirma este hecho, puesto que sus resultados apuntaron a una significancia entre bajo rendimiento académico y edad. ${ }^{1}$

En nuestra población hubo una frecuencia de tener sintomatología compatible con TDAH del 27,5\%. En el estudio realizado por Iván Fajardo et al se observó una frecuencia del 10,7\% de padecer dichos síntomas. ${ }^{12}$ En la tesis de Villaseñor Valadez también se vio reflejado un bajo porcentaje de alumnado que padece TDAH, correspondiente al $15,1 \%$ de un total de 466 estudiantes. ${ }^{13}$ En el análisis multivariado del presente estudio se obtuvo una asociación significativa del TDAH con el bajo rendimiento académico, de igual manera que en el estudio realizado por Curay Roque. ${ }^{1}$

En lo que corresponde a la ansiedad, los estudiantes mostraron tener un alto porcentaje, $75,4 \%$ de los estudiantes. Altos porcentajes también se hallaron en el estudio realizado en Costa Rica, donde se evidenció que la ansiedad en sus estudiantes estuvo presente en el $67,8 \%$ de ellos. ${ }^{14}$ Caso contrario, en los estudios realizados en Medellín, en el 2019, solo el 26,5\% de estudiantes del área de la salud presentó ansiedad. ${ }^{15}$ En el artículo de Pimienta et al, donde los estudiantes fueron evaluados con el STAI (State-Trait Anxiety Inventory) para ansiedad, las puntuaciones fueron significativamente mayores en el curso de enfermería que en el de tecnología médica al relacionarlos con el bajo rendimiento académico. ${ }^{16}$ También se encontró asociación de ansiedad con bajo rendimiento académico en el estudio de Roque, para los estudiantes de medicina humana de una universidad privada. ${ }^{1}$

La variable depresión se halló en el 78,8\% de los estudiantes. En un estudio de la Universidad Autónoma de México, el $57 \%$ de los estudiantes presentó sintomatología depresiva. ${ }^{17}$ En el caso de los estudiantes de la Universidad Santa Marta ocurrió en el $42,2 \%$ de alumnos. ${ }^{18}$ En un estudio realizado en Colombia, el 30,15\% de los estudiantes de medicina, de la Universidad de Antioquia, presentó sintomatología compatible con depresión y el $45,9 \%$ tuvo bajo rendimiento académico y depresión. ${ }^{15}$ En otro estudio realizado en Lima, mencionaron una asociación significativa entre depresión y bajo rendimiento académico en estudiantes de medicina humana, en el 2017. ${ }^{9}$ También, en un estudio realizado en el departamento de La Libertad, en 2019, nos encontramos con la asociación de las variables ya mencionada. ${ }^{1}$

En lo que compete a la variable sexo, el porcentaje de mujeres con bajo rendimiento fue de $71,9 \%$; sin embargo, en este estudio no se encontró asociación significativa. Un estudio, realizado en Trujillo, nos mostró una asociación del sexo femenino con el bajo rendimiento académico. ${ }^{1}$ Otros nos mencionan que el sexo masculino fue el que se relacionó con el bajo rendimiento académico, donde además se relacionó a consumo de $\operatorname{drogas},{ }^{20}$ y en el artículo sobre procrastinación académica y ansiedad en estudiantes de ciencias de la salud, en una Universidad de Lima Norte, mostró también que ser varón y tener un bajo rendimiento académico fue una asociación estadísticamente significativa. ${ }^{21}$

El ciclo con más bajo rendimiento fue el $11^{\circ}$ ciclo, aunque sin asociaciones significativas. Lo mismo ocurrió en el estudio realizado por Merchán, donde encuestaron a estudiantes de medicina desde $4^{\circ}$ ciclo a $10^{\circ}$ ciclo, y no hubo valores estadísticos de significancia. ${ }^{22}$

Los estudiantes de medicina, al tener que adquirir basta información, tienen una mejor forma de organizarse, generalmente, y esto es mediante métodos de estudios. Así, observamos que el $92,3 \%$ de los estudiantes poseía algún método. En el análisis bivariado no se halló relación significativa con el bajo rendimiento. En un estudio realizado en Cuba, en estudiantes de medicina, tampoco se encontró relación con las variables mencionadas. ${ }^{22}$ Mientras, sucedió lo contrario en la investigación que realizó García et al, donde aparte de no tener buen método de estudio, el divorcio $\mathrm{y}$ altos niveles de estrés fueron asociados al bajo rendimiento académico. El trabajo de investigación realizado por Manuel Chilca Alva, indica que existe relación de las variables antes descritas, por lo cual recomiendan que, si estos métodos mejoraran, incrementaría el rendimiento académico. ${ }^{24}$

De los estudiantes encuestados, el $85,3 \%$ solo estudiaba y $14,7 \%$ estudiaba y trabajaba. En el análisis bivariado, aquellos alumnos que estudiaban y trabajaban y con bajo rendimiento académico fueron el $90 \%$, teniendo asociación significativa. En un trabajo de investigación realizado Colombia, sobre prevalencia y factores asociados a sintomatología depresiva y ansiedad rasgo en estudiantes universitarios del área de la salud, nos mostró que el 20,6\% trabajaba y estudiaba, un porcentaje similar al de nuestro estudio. ${ }^{15}$

También se encontró que el $88,6 \%$ de los estudiantes viven con sus familias, mientras que un pequeño porcentaje vive solo. Cuando se asoció con la variable de bajo rendimiento académico no se halló significancia. Lo mismo sucedió en el trabajo de investigación de Diana Curay. ${ }^{1}$

En lo que respecta al consumo de drogas, encontramos que los estudiantes de medicina humana niegan en su mayoría haber tenido contacto con estas sustancias alguna vez en su vida, lo que correspondió al 63,4\%, y en el análisis bivariado no se halló asociación con el bajo rendimiento; sin embargo, en el estudio realizado por Garrido et al se halló una asociación significativa del consumo de drogas con el bajo rendimiento, siendo dos veces mayor. ${ }^{25}$ En el artículo realizado por Merchán, Romero y Alameda, sobre consumo de sustancias psicoactivas, inteligencia emocional y rendimiento académico en una muestra de estudiantes universitarios, nos mostraron una relación significativa entre aquellos estudiantes consumidores de drogas con un bajo nivel académico. ${ }^{26}$ El estudio realizado por Sotelo reveló que consumir drogas, en este caso, anfetaminas, está relacionado con ser varón y presentar bajo rendimiento académico. ${ }^{20}$ 
Aquellos estudiantes que consumen alcohol y fuman fueron el $27,5 \%$ y $9,2 \%$, respectivamente, de los cuales, bajo rendimiento tuvieron el $70,7 \%$ y $88,00 \%$ en las correspondientes variables; sin embargo, no se encontró asociación significativa. En el artículo de Garrido et al se halló que el consumo de alcohol fue de $89,9 \%$ y de tabaco $36,2 \%$, cifras muchas más altas que en el presente estudio. ${ }^{25}$ En otro estudio realizado en España se encontró asociación significativa de consumo de tabaco con bajo rendimiento académico. ${ }^{26}$

Los resultados obtenidos en nuestro estudio deben conducirnos a la aplicación de estrategias para la prevención ante los trastornos descritos. Asimismo, habría que realizar diversas pruebas (tests) más especializadas a los ingresantes para poder identificar problemas a nivel psicológico/psiquiátrico, obteniendo un diagnóstico precoz y así ayudarlos desde un inicio con la pedagogía necesaria, $\mathrm{y}$ atendidos por el profesional que requiera el alumno.

Es recomendable implementar charlas educativas tanto a docentes como al alumnado sobre temas de salud mental para guiarlos y puedan reconocer los problemas y brinda el apoyo requerido oportunamente.

En conclusión, la proporción de bajo rendimiento académico en alguna asignatura cursada en el periodo 2020II en la población encuestada fue alta. Ansiedad y depresión fueron las variables con mayor prevalencia; y, se encontró asociación entre tener sintomatología compatible con TDAH y mayor edad con el bajo rendimiento académico.

\section{REFERENCIAS BIBLIOGRÁFICAS}

I. Curay Roque DK. Trastorno de déficit de atención e hiperactividad como factor de bajo rendimiento en estudiantes de medicina humana. Tesis de bachiller en medicina. Universidad Privada Antenor Orrego, Trujillo; 2019. URL disponible en: https://hdl. handle.net/20.500.12759/5253

2. Pego-Pérez E Rubén, Río-Nieto $M^{a}$ del Carmen del, Fernández Isaac, Gutiérrez-García Emilio. Prevalencia de sintomatología de ansiedad y depresión en estudiantado universitario del Grado en Enfermería en la Comunidad Autónoma de Galicia. Ene. 2018; 12(2): 225. URL disponible en: http://scielo.isciii.es/scielo.php?script=sci_ arttext\&pid=SI 988-348X2018000200005\&lng=es.

3. Ortiz Castillo JA. Relación de la Ansiedad y Depresión sobre el rendimiento académico en estudiantes de medicina humana. Psiquiatría y Salud Mental. 2016;XXXIII(3/4):I23-I3I.

4. De San Jorge Cárdenas X, Beverido Sustaeta P, Salas García B, Cruz Juárez A, Roa Cubaque MA, Rubiano Díaz GH. Drogas y rendimiento académico en estudiantes del área de salud de dos universidades latinoamericanas. Pensando Psicol. 2017; 13(22): 5 I-60. Disponible en: https://revistas.ucc.edu.co/index.php/pe/article/view/I988

5. American Psychiatric Association. Manual diagnóstico y estadístico de los trastornos mentales DSM-5. $5^{\circ}$ ed. Editorial Médica Panamericana; 2014.

6. Coryel, William. Trastornos depresivos - Trastornos psiquiátricos. Manual MSD versión para profesionales. URL disponible en: https://www.msdmanuals.com/es-pe/professional/trastornospsiqui\%C3\%A I tricos/trastornos-del-estado-de-\%C3\%AInimo/ trastornos-depresivos

7. Sistema de calificaciones del Perú en relación a los sistemas de otros países tabla de equivalencias. Universidad Ricardo Palma. URL disponible en: https://www.urp.edu.pe/pdf/id//25/4/n/

8. The World Health Organization Adult ADHD Self-Report Scale (ASRS): A short screening scale for use in the general population.
Psychological Medicine. 2005;35, 245-256. URL disponible en: https:// pubmed.ncbi.nlm.nih.gov//584/682/

9. Goldberg D, Bridges K, Duncan-Jones P, et al. Detecting anxiety and depression in general medical settings. Br Med J. 1988;97:897-99. URL disponible en: https://pubmed.ncbi.nlm.nih.gov/3/40969/

10. Encuestas escolares sobre el uso indebido de drogas: programa mundial de evaluación del uso indebido de drogas. módulo 3 del manual. Nueva York: Naciones Unidas; 2003.

II. Brito-Jiménez IT, Palacio-Sañudo J. Calidad de vida, desempeño académico y variables sociodemográficas en estudiantes universitarios de Santa Marta-Colombia. Duazary. 2016;13(2):।3341.

12. Fajardo Villeda IA. Cribado de trastorno por déficit de atención e hiperactividad en estudiantes de medicina. Rev Cub. 2020;4(I): I-6. URL disponible en: https://revistacunori.com/index.php/cunori/ article/view/I03

13. Valadez VDV. Prevalencia del TDAH en adultos universitarios jóvenes. Neuropsicología clínica UNAM, 8 de octubre de 2018. XII Congreso de Posgrado de Psicología. URL disponible en: https:// cuved.unam.mx/divulgacion/index.ph p/CPMDP/ XIICPPUNAM20I8/paper/view/4I8

14. Delgado Monge IC, Espinoza González J, Fonseca Castro J. Ansiedad matemática en estudiantes universitarios de Costa Rica y su relación con el rendimiento académico y variables sociodemográficas. Propósitos y Representaciones. 20I7; 5(I):275-324. http://revistas.usil.edu.pe/index.php/pyr/article/view/I48

15. Caro Y, Trujillo S, Trujillo N. Prevalencia y factores asociados a sintomatología depresiva y ansiedad rasgo en estudiantes universitarios del área de la salud. Psychol Av Discip. 20 I 9; I 3(I):4 I-52.

16. Castillo Pimienta C, Chacón de la Cruz T, Díaz-Véliz G, Castillo Pimienta C, Chacón de la Cruz T, Díaz-Véliz G. Ansiedad y fuentes de estrés académico en estudiantes de carreras de la salud. Investig En Educ Médica. diciembre de 2016;5(20):230-7.

17. Serrano-Barquín C, Rojas-García A, Ruggero. Depresión, ansiedad y rendimiento académico en estudiantes universitarios. Revista Intercontinental de Psicología y Educación. 2013;15(I):47-60. URL disponible en: https://www.redalyc.org/pdf/802/80225697004.pdf

18. Franco Mejía, Gutiérrez Agudelo S, Perea E. Asociación entre depresión y bajo rendimiento académico en estudiantes universitarios. Psicogente 20II;|4(25):67-75. URL disponible en: https://www.redalyc.org/articulo.oa?id=497552358007

19. Márquez L, Luis J. Depresión y rendimiento académico de los estudiantes universitarios de la Facultad de Medicina de la Universidad Científica del Sur en el periodo 2017. Tesis de maestría. Universidad José Carlos Mariátegui. Moquegua;20 I 7.URL disponible en: https://repositorio.ujcm.edu.pe/handle/20.500.12819/308

20. Sotelo Ale, AM. Factores socioeducativos asociados al consumo de anfetaminas en universitarios de Latinoamérica. Tesis para optar el título profesional de médico cirujano, Escuela Académico Profesional de Medicina Humana, Universidad Continental, Huancayo, Perú; 2020. URL disponible en: https://repositorio. continental.edu.pe/handle/20.500.12394/7335

21. Gil-Tapia L, Botello-Príncipe V. Procrastinación académica y ansiedad en estudiantes de Ciencias de la Salud de una Universidad de Lima Norte. CASUS Rev Investig Casos En Salud. 2018;3(2):89-96.

22. Merchán-Galvis ÁM, López HFS, Robledo JEG, Patiño JNO, Guerrero CCA, Martínez JJ. Estudio de casos y controles de factores relacionados con el rendimiento académico en estudiantes de medicina. Rev Cuba Educ Médica Super. 2017;3 I (3): I0I-9.

23. García Gascón A, Del Toro Añel AY, Cisneros Prego E, Querts Méndez $O$, Cascaret Soto $X$. Algunas variables psicosociales asociadas al bajo rendimiento académico en estudiantes de primer año de medicina. MEDISAN. abril de 20I7;2I (4):433-9.

24. Chilca Alva ML. Autoestima, hábitos de estudio y rendimiento académico en estudiantes universitarios. Propósitos Represent. 20I7;5(I):7I-I27.

25. Garrido-González I, Bugarín-González R, Machín-Fernández AJ. Consumo de drogas en estudiantes de enfermería. Enferm Clínica. 2016;26(3): I74-80. 
26. Merchán Clavellino A, Moreno AF, Alameda J. Consumo de sustancias psicoactivas, inteligencia emocional y rendimiento académico en una muestra de estudiantes universitarios. Rev Española Drogodepend. 2017;42:21-34.

\section{CORRESPONDENCIA:}

Jennifer M. Otero-Zapata

jenn8otero@gmail.com
Fecha de recepción: 21-08-2021.

Fecha de aceptación: 06-09-2021.

Conflicto de interés: ninguno, según los autores.

Contribuciones de autoría: Los autores participaron en la génesis de la idea, diseño de proyecto e interpretación de datos, análisis de resultados y preparación del manuscrito del presente trabajo de investigación.

Financiamiento:Autofinanciado 